\title{
Association of vascular endothelial growth factor polymorphisms with polycystic ovarian syndrome risk: a meta-analysis
}

\author{
Jiahui Zhao' ${ }^{1}$ Da Li², Huaiyun Tang ${ }^{1}$ and Lisha Tang ${ }^{1 *}$
}

\begin{abstract}
Background: Polycystic ovarian syndrome (PCOS) is a multi-gene hereditary disorder caused by the interaction of certain gene variation with environmental factors. Previous studies have shown that vascular endothelial growth factor (VEGF) gene polymorphisms are associated with the risk of polycystic ovarian syndrome. However, the results of these studies remain controversial. We performed the present meta-analysis aiming to further investigate the potential relationship between VEGF polymorphisms and susceptibility to PCOS.

Methods: The following databases were systematically searched: PubMed, EMBASE, Web of Science (WOS), China National Knowledge Infrastructure (CNKI), and Wanfang Databases. The correlation between VEGF polymorphisms and PCOS risk was assessed by calculating pooled odds ratios (ORs) and their $95 \%$ confidence intervals (95\% Cls). Subgroup analyses stratified by ethnicity and source of control were also conducted. Besides, trial sequential analysis (TSA) was done to verify the reliability of the pooled results.

Results: 10 relevant case-control studies were incorporated in this meta-analysis, involving 1347 PCOS cases and 1378 controls. The VEGF rs2010963 polymorphism was associated with decreased PCOS risk in the whole population and the Asian populations. The VEGF rs3025039 polymorphism was associated with decreased PCOS susceptibility and the Asian populations, but increased risk of PCOS was observed among the Caucasian populations. In addition, the results of trial sequential analysis (TSA) showed the negative correlation between rs2010963 and PCOS risk, obtained by our meta-analysis, was stable and reliable.

Conclusion: Overall, different VEGF gene polymorphisms may exert different effects on PCOS susceptibility. The VEGF rs2010963 polymorphism decreases PCOS susceptibility in both the whole population and the Asian populations, and VEGF rs3025039 polymorphism causes lower PCOS susceptibility in the whole population and the Asian populations but higher in the Caucasian populations.
\end{abstract}

Keywords: VEGF, Polymorphism, Meta-analysis, Polycystic ovarian syndrome

\footnotetext{
* Correspondence: 540660190@qq.com

${ }^{1}$ Department of Reproductive Medicine, Lianyungang Maternal and Child Health Hospital, NO.669 Qindongmen Road, Lianyungang 222001, Jiangsu Province, China

Full list of author information is available at the end of the article
}

(C) The Author(s). 2020 Open Access This article is licensed under a Creative Commons Attribution 4.0 International License, which permits use, sharing, adaptation, distribution and reproduction in any medium or format, as long as you give appropriate credit to the original author(s) and the source, provide a link to the Creative Commons licence, and indicate if changes were made. The images or other third party material in this article are included in the article's Creative Commons licence, unless indicated otherwise in a credit line to the material. If material is not included in the article's Creative Commons licence and your intended use is not permitted by statutory regulation or exceeds the permitted use, you will need to obtain permission directly from the copyright holder. To view a copy of this licence, visit http://creativecommons.org/licenses/by/4.0/ The Creative Commons Public Domain Dedication waiver (http://creativecommons.org/publicdomain/zero/1.0/) applies to the data made available in this article, unless otherwise stated in a credit line to the data. 


\section{Introduction}

Polycystic ovarian syndrome (PCOS) is one of the most common endocrine diseases with complex etiology and pathogenesis, with a prevalence of up to $10 \%$ in reproductive age women $[1,2]$. It is generally characterized by high androgen, long-term persistent anovulation, and ovarian polycystic changes. Typical clinical manifestations include menstrual disorders, infertility, hypertrichosis, acne, obesity, acanthosisnigricans, and polycystic changes in the ovary detected by ultrasound [3]. PCOS is often associated with a variety of metabolic diseases involving patients with varying degrees of insulin resistance, hyperinsulinemia and hyperlipidemia [4]. These metabolic disorders make PCOS patients susceptible to type 2 diabetes, as well as cardiovascular and cerebrovascular diseases [5]. PCOS accounts for approximately $75 \%$ of anovulatory infertility $[6,7]$. The early abortion rate in PCOS patients is as high as 30 to $50 \%$, and the occurrence of recurrent pregnancy loss is also up to $50 \%$, thus seriously affecting the outcome of pregnancy $[8,9]$. The pathogenesis of PCOS, complicated and multifactorial, has not yet been fully elucidated. Currently, it is generally believed that PCOS may be a multi-gene hereditary disorder caused by the interaction of certain genes with environmental factors. Mounting evidence exists that certain genetic polymorphisms play an important role in the pathogenesis of PCOS [10-13].

Vascular endothelial growth factor (VEGF), a homodimeric glycoprotein hormone first purified by Ferrara et al. from the bovine pituitary follicular stellate cells in vitro, is the most active angiogenic factor ever known, which can maintain the differentiation state of vascular endothelial cells and improve microvascular permeability, and play a powerful regulatory role in the occurrence and development of various diseases [14, 15]. It is closely related to the pathogenesis of PCOS, and studies have reported a significant rise in serum level of VEGF in the PCOS patients [16-18]. Additionally, the overexpression of VEGF gene in the polycystic ovarian stroma also reveals the critical role of this gene in the pathophysiology of PCOS [19].

Recent researches have focused on the correlation between VEGF gene polymorphism and PCOS, with rs2010963 (G > C), rs833061 ( $>$ > C), rs3025039 $(\mathrm{C}>\mathrm{T})$, rs699947 (A > C), and rs1570360 (A > G) extensively investigated for their potential relationship to PCOS risk [20-29]. However, due to different ethnicities and small study sample size, evidence obtained from the different studies remains inconclusive and their results are even controversial. Therefore, we undertook this metaanalysis to explore more comprehensively the relationship between VEGF gene polymorphism and the risk of PCOS by combining existing research results.

\section{Materials and methods}

\section{Literature search}

After formulating the search strategy, we systematically searched the databases of PubMed, EMBASE, Web of Science (WOS), Chinese National Knowledge Infrastructure (CNKI) and Wan Fang to collect potentially eligible case-control studies related to VEGF gene polymorphisms and the risk of PCOS. The last retrieval was conducted on October 1, 2019. The search terms were as follows: ("VEGF" OR "vascular endothelial growth factor") and ("PCOS" OR "Polycystic ovary syndrome") and ("mutation" OR "variant" OR "polymorphism" OR "genotype" OR "allele" OR "gene" OR "snp"). To avoid the omission of relevant research, references in the eligible literature after retrieval were also browsed and manually retrieved.

\section{Inclusion and exclusion criteria}

The studies included in this meta-analysis must meet the following criteria: (a) Case-control studies covered the relationship between VEGF gene polymorphisms and risk of PCOS. (b) The sample size, genotype distribution, and genotype frequency of the case and control groups were directly provided in the document, or the literature contained available original data that could be converted into OR value and $95 \% \mathrm{CI}$ for the case and control groups. (c) The diagnosis of PCOS patients in the case group must conform to one of the following three diagnostic criteria: NIH, Rotterdam, and AE-PCOS Society [30-32] (d). The inclusion criteria for the control group were clear and definite. (e) If multiple articles based on the same case and control population were published by the same author or research center, only relevant researches in the latest publication time would be incorporated.

Studies were excluded on the basis of the following criteria: (a) There was no clear definition of polycystic ovary syndrome in the literature, and no clear diagnostic criteria were available for enrolled patients in the case group. (b) The specific genotype data of the case group and the control group were not provided, or the genotype frequency of the case group and the control group could not be calculated by the original data in the literature. (c) Conference papers, case reports, literature reviews or animal researches were ruled out.

\section{Data extraction}

Two reviewers (Jiahui Zhao and Da $\mathrm{Li}$ ) read screened independently the eligible relevant literature according to the inclusion and exclusion criteria. When the two researchers disagreed about whether or not a study should be included, they would try to resolve it through discussion. If they could not reach a consensus, the final decision would be made by a third researcher (Lisha Tang). 
For the included literature after screening, the following data were mainly extracted: first author, publication year, nationality, ethnicity, PCOS diagnostic criteria adopted, inclusion criteria of the control group, source of control, genotyping method, single nucleotide polymorphisms (SNPs) investigated in the study, sample size and genotype distribution of the case group and the control group.

\section{Quality assessment}

The literature quality was evaluated by NewcastleOttawa Scale (NOS) [33]. The NOS evaluation criterion of case-control study included 9 items (1 point for each item, 9 points in total). The specific scores involved: (1) selection of the case group and control group (4 points) based on appropriate definition and diagnosis of the case, representativeness of the case, selection of control, and definition of control; (2) comparability between the case group and control group (2 points); and (3) exposure evaluation of the case group and control group (4 points). If the study satisfied the evaluation score at 7 points or above, the quality of the literature was thought to be high; if the research evaluation score ranged 4-6, the quality of the literature was medium; and if the score wass $<4$, the literature quality was considered low.

\section{Statistical analysis}

Meta-analysis was mainly performed using Revman 5.3 software by the Cochrane Collaboration. The strength of association between VEGF polymorphisms and susceptibility of PCOS was evaluated by calculating Odds ratios (OR) and its 95\% confidence intervals (CIs) under five gene comparison models. For example, the correlation between rs2010963 ( $\mathrm{G}>\mathrm{C}$ ) polymorphism and PCOS risk was measured and assessed under the following models: allele model (C vs. G), dominant model $(\mathrm{GC}+\mathrm{CC}$ vs. $\mathrm{GG}$ ), heterozygous model (GC vs. GG), recessive model (CC vs. GC+ GG) and homozygous model (CC vs. GG). The heterogeneity between studies was quantitatively determined by $\mathrm{I}^{2}$ statistic. If $\mathrm{I}^{2}$ was lower than $50 \%$, which indicated that the heterogeneity was low, the fixed-effect model would be applied for meta-analysis. If statistical heterogeneity between studies existed $\left(\mathrm{I}^{2}>50 \%\right)$, the source of heterogeneity would be further analyzed, and the random effect model would be adopted. In addition to overall analysis, subgroup analyses stratified by ethnicity

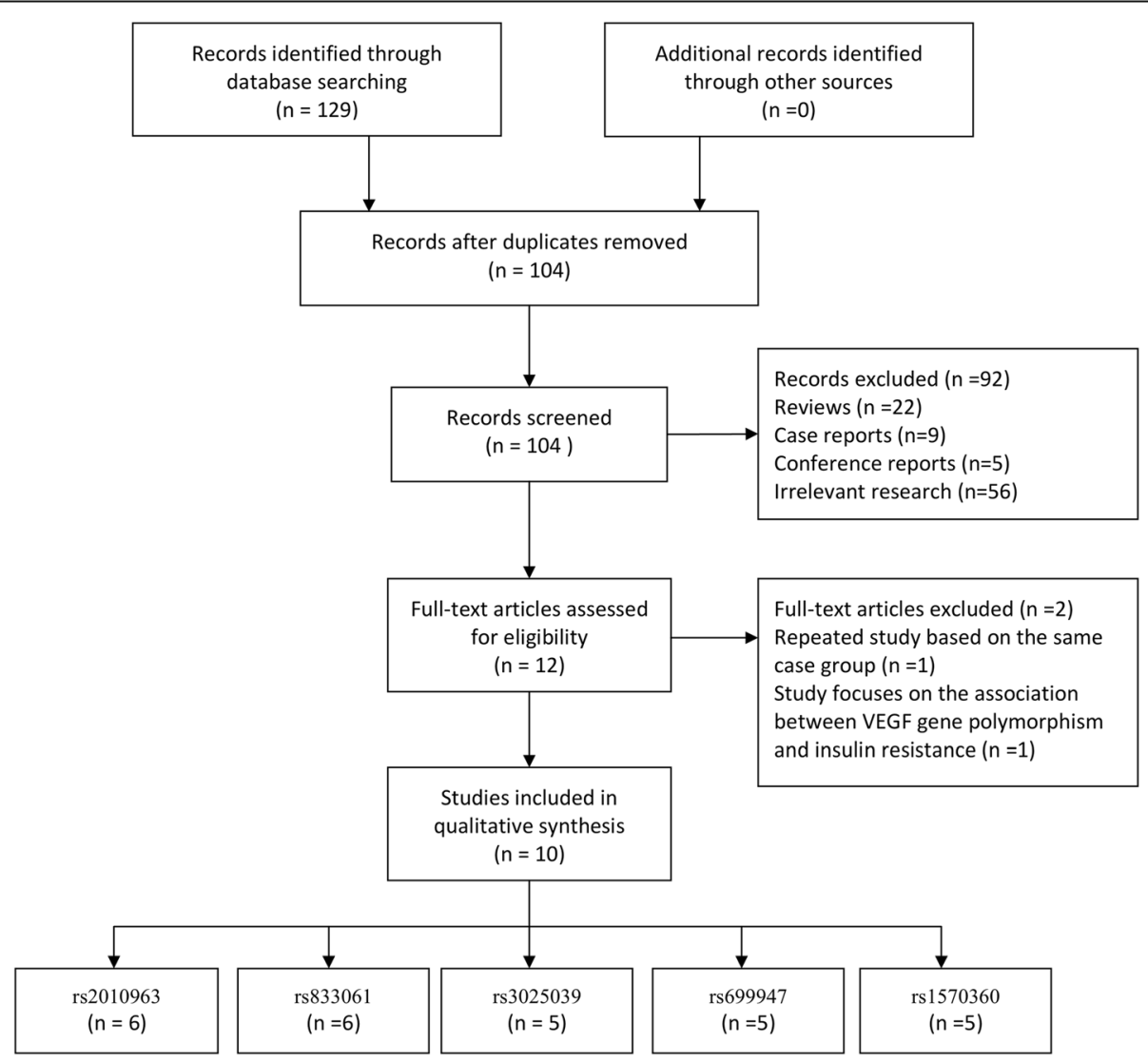

Fig. 1 Flow diagram of selection and inclusion process 
and source of control were also performed. Since all of the included literature adopted the Rotterdam diagnostic criteria for the enrolled patients of case groups, there was no subgroup analyses based on different diagnostic criteria. If there was only one article in a subgroup, no separate analysis would be performed.

The possible publication bias was first evaluated by observing the symmetry of the funnel plots. The Stata14.0 software (STATA Corporation, College Station, TX) was further applied to perform the Begg's and Egger's test procedures to assess potential publication bias. The genotype distribution of the control group was calculated by $x^{2}$ test to ascertain whether the control group was consistent with Hardy-Weinberg equilibrium (HWE).

Traditional meta-analysis methods have no way to assess the adequacy of the overall sample size of the included studies. When the overall sample size is not sufficient, the final pooled results obtained are not reliable enough, especially the positive results obtained by meta-analysis. Statistical results based on insufficient sample size increase the likelihood of false positive results (Type 1 error). Therefore, in the present study, we adopted trial sequential analysis (TSA) method to determine whether the overall sample size of the included studies was sufficient and whether the overall results

Table 1 Characteristics of included studies

\begin{tabular}{|c|c|c|c|c|c|c|c|c|c|}
\hline Author & Year & Region & Ethnicity & $\begin{array}{l}\text { PCOS } \\
\text { Diagnostic } \\
\text { criteria }\end{array}$ & Inclusion criteria for the control group & $\begin{array}{l}\text { Control } \\
\text { source }\end{array}$ & $\begin{array}{l}\text { Genotyping } \\
\text { method }\end{array}$ & SNP & NO \\
\hline $\begin{array}{l}\text { Almawi } \\
{[20]}\end{array}$ & 2016 & Bahrain & Caucasian & Rotterdam & $\begin{array}{l}\text { age-and ethnically-matched; testosterone levels were } \\
\text { within range }(0.4-3.5 \mathrm{nmol} / \mathrm{L}) \text { in the menstrual cycle } \\
\text { follicular phase; no metabolic or endocrine diseases; } \\
\text { BMI were between } 18 \text { and } 45 \mathrm{~kg} / \mathrm{m}^{2} \text {; without recent ill- } \\
\text { ness; without treatment may affect carbohydrate me- } \\
\text { tabolism or hormonal levels within } 3 \text { months; without } \\
\text { using anti-hypertensive, oral contraceptive, anti- } \\
\text { inflammatory and lipid-lowering drugs }\end{array}$ & PB & RT-PCR & $\begin{array}{l}\text { rs2010963 } \\
\text { rs833061 } \\
\text { rs3025039 } \\
\text { rs699947 } \\
\text { rs1570360 }\end{array}$ & 8 \\
\hline Bao [21] & 2019 & India & Asian & Rotterdam & $\begin{array}{l}\text { age-and ethnically-matched; testosterone levels were } \\
\text { within range }(0.4-3.5 \mathrm{nmol} / \mathrm{L}) \text { in the menstrual cycle } \\
\text { follicular phase; no metabolic or endocrine diseases; } \\
\text { BMI were between } 18 \text { and } 45 \mathrm{~kg} / \mathrm{m}^{2} \text {; without recent ill- } \\
\text { ness; without using anti-hypertensive, oral contracep- } \\
\text { tive, anti-inflammatory and lipid-lowering drugs }\end{array}$ & PB & RT-PCR & $\begin{array}{l}\text { rs2010963 } \\
\text { rs833061 } \\
\text { rs3025039 } \\
\text { rs699947 } \\
\text { rs1570360 }\end{array}$ & 6 \\
\hline $\begin{array}{l}\text { Ben Salem } \\
{[22]}\end{array}$ & 2016 & Tunisia & Caucasian & Rotterdam & $\begin{array}{l}\text { women with regular menstrual cycles and no } \\
\text { evidence of hirsutism, acne, alopecia, or } \\
\text { endocrinopathies; without treatment may affect } \\
\text { carbohydrate metabolism or hormonal levels within } 3 \\
\text { months }\end{array}$ & PB & RT-PCR & $\begin{array}{l}\text { rs833061 } \\
\text { rs3025039 } \\
\text { rs699947 } \\
\text { rs1570360 }\end{array}$ & 7 \\
\hline Ding [28] & 2009 & China & Asian & Rotterdam & $\begin{array}{l}\text { women with regular menstrual cycles; with normal } \\
\text { ovarian morphology; without treatment may affect } \\
\text { carbohydrate metabolism or hormonal levels within } 3 \\
\text { months; no metabolic or endocrine diseases; }\end{array}$ & $H B$ & $P C R$ & rs3025039 & 6 \\
\hline $\begin{array}{l}\text { Gomes } \\
{[23]}\end{array}$ & 2019 & Brazil & Caucasian & Rotterdam & $\begin{array}{l}\text { age- matched; fertile women with no history of } \\
\text { hyperandrogenism, menstrual dysfunction, infertility, } \\
\text { or sonographic signal of PCOS; without using } \\
\text { contraceptives, antiandrogens, statins, glucocorticoids } \\
\text { or infertility medications within } 6 \text { months }\end{array}$ & PB & $\begin{array}{l}\text { PCR-RFLP } \\
\text { (rs699947) } \\
\text { RT-PCR } \\
\text { (rs1570360) }\end{array}$ & $\begin{array}{l}\text { rs699947 } \\
\text { rs1570360 }\end{array}$ & 6 \\
\hline $\begin{array}{l}\text { Guruvaiah } \\
{[24]}\end{array}$ & 2014 & India & Asian & Rotterdam & $\begin{array}{l}\text { fertile women with regular menstrual cycles and had } \\
\text { a successful pregnancy record; with normal ovarian } \\
\text { morphology confirmed by ultrasound }\end{array}$ & PB & $P C R$ & $\begin{array}{l}\text { rs2010963 } \\
\text { rs833061 }\end{array}$ & 7 \\
\hline $\begin{array}{l}\text { Huang } \\
{[25]}\end{array}$ & 2019 & China & Asian & Rotterdam & $\begin{array}{l}\text { women with normal menstrual cycles and had at } \\
\text { least one successful pregnancy history; without } \\
\text { endocrine disorders, family history of diabetes or } \\
\text { sonographic signs of PCOS }\end{array}$ & $H B$ & PCR-LDR & $\begin{array}{l}\text { rs } 2010963 \\
\text { rs833061 }\end{array}$ & 7 \\
\hline Lee [26] & 2008 & Korea & Asian & Rotterdam & $\begin{array}{l}\text { BMI-matched; with regular menstrual cycles; with } \\
\text { normal ovarian morphology confirmed by ultrasound }\end{array}$ & PB & PCR & $\begin{array}{l}\text { rs2010963 } \\
\text { rs3025039 }\end{array}$ & 6 \\
\hline Li [29] & 2014 & China & Asian & Rotterdam & fertile women with regular menstrual cycles & $\mathrm{HB}$ & PCR-RFLP & rs1570360 & 6 \\
\hline Vural [27] & 2009 & Turkey & Caucasian & Rotterdam & $\begin{array}{l}\text { fertile women without hyperandrogenism, history of } \\
\text { menstrual dysfunction, infertility or sonographic signs } \\
\text { of PCOS. }\end{array}$ & PB & PCR & $\begin{array}{l}\text { rs2010963 } \\
\text { rs833061 } \\
\text { rs699947 }\end{array}$ & 6 \\
\hline
\end{tabular}


obtained were reliable. Trial sequential analysis (TSA) was performed by using the TSA v0.9.5.10 Beta software [34]. For the positive results obtained from metaanalysis, TSA method was applied to analyze the reliability of the results and examine whether the sample size was sufficient. If the cumulative $\mathrm{Z}$ curve crossed trial sequential monitoring boundary, the result was considered stable and reliable. The sufficiency of overall sample size was estimated based on whether the cumulative Z-curve exceeded the required information size (RIS).

\section{Ethical approval}

The meta-analysis was performed by summarizing data from previous studies. Therefore, no ethical approval was required.

Table 2 Genotype distribution and HWE

\begin{tabular}{|c|c|c|c|c|c|c|c|c|c|c|}
\hline \multirow[t]{2}{*}{ SNP } & \multirow[t]{2}{*}{ Author } & \multicolumn{2}{|c|}{ Sample size } & \multicolumn{3}{|c|}{ Case Genotype } & \multicolumn{3}{|c|}{ Control genotype } & \multirow{2}{*}{$\begin{array}{l}\mathrm{HWE} \\
\mathrm{Y} / \mathrm{N}(\boldsymbol{p})\end{array}$} \\
\hline & & Case & Control & $\overline{G G}$ & GC & $\mathrm{CC}$ & $\overline{G G}$ & GC & CC & \\
\hline \multirow[t]{6}{*}{ rs2010963 } & Almawi & 382 & 393 & 183 & 142 & 57 & 161 & 190 & 42 & 0.45 \\
\hline & Bao & 55 & 52 & 28 & 11 & 16 & 26 & 12 & 14 & 0.47 \\
\hline & Guruvaiah & 126 & 130 & 70 & 46 & 10 & 52 & 59 & 19 & 0.73 \\
\hline & Huang & 118 & 130 & 60 & 45 & 13 & 47 & 64 & 19 & 0.71 \\
\hline & Lee & 134 & 100 & 46 & 60 & 26 & 34 & 45 & 20 & 0.47 \\
\hline & Vural & 137 & 155 & 90 & 44 & 3 & 112 & 39 & 4 & 0.78 \\
\hline \multirow[t]{2}{*}{ SNP } & Author & \multicolumn{2}{|c|}{ Sample size } & \multicolumn{3}{|c|}{ Case Genotype } & \multicolumn{3}{|c|}{ Control genotype } & HWE \\
\hline & & Case & Control & $\mathrm{TT}$ & $\mathrm{TC}$ & $\mathrm{CC}$ & $\mathrm{TT}$ & $\mathrm{TC}$ & $\mathrm{CC}$ & $\mathrm{Y} / \mathrm{N}(p)$ \\
\hline \multirow[t]{6}{*}{ rs833061 } & Almawi & 382 & 393 & 130 & 174 & 78 & 132 & 190 & 71 & 0.91 \\
\hline & Bao & 55 & 52 & 32 & 9 & 14 & 24 & 11 & 17 & 0.06 \\
\hline & Ben Salem & 118 & 150 & 33 & 55 & 30 & 42 & 76 & 32 & 0.82 \\
\hline & Guruvaiah & 126 & 130 & 40 & 59 & 27 & 33 & 72 & 25 & 0.20 \\
\hline & Huang & 118 & 130 & 63 & 45 & 10 & 80 & 42 & 8 & 0.44 \\
\hline & Vural & 137 & 155 & 55 & 64 & 18 & 52 & 74 & 29 & 0.77 \\
\hline \multirow[t]{2}{*}{ SNP } & Author & \multicolumn{2}{|c|}{ Sample size } & \multicolumn{3}{|c|}{ Case Genotype } & \multicolumn{3}{|c|}{ Control genotype } & HWE \\
\hline & & Case & Control & $\mathrm{CC}$ & $\mathrm{CT}$ & $\mathrm{TT}$ & $\mathrm{CC}$ & $\mathrm{CT}$ & $\mathrm{TT}$ & $\mathrm{Y} / \mathrm{N}(p)$ \\
\hline \multirow[t]{5}{*}{ rs3025039 } & Almawi & 382 & 393 & 296 & 81 & 5 & 318 & 68 & 7 & 0.78 \\
\hline & Bao & 55 & 52 & 31 & 9 & 15 & 24 & 11 & 17 & 0.10 \\
\hline & Ben Salem & 118 & 150 & 89 & 27 & 2 & 127 & 19 & 4 & 0.005 \\
\hline & Ding & 80 & 84 & 45 & 35 & 0 & 54 & 30 & 0 & 0.04 \\
\hline & Lee & 134 & 100 & 89 & 35 & 4 & 59 & 31 & 10 & 0.06 \\
\hline \multirow[t]{2}{*}{ SNP } & Author & \multicolumn{2}{|c|}{ Sample size } & \multicolumn{3}{|c|}{ Case Genotype } & \multicolumn{3}{|c|}{ Control genotype } & HWE \\
\hline & & Case & Control & $A A$ & $A C$ & $\mathrm{CC}$ & $A A$ & $\mathrm{AC}$ & $\mathrm{CC}$ & $\mathrm{Y} / \mathrm{N}(p)$ \\
\hline \multirow[t]{5}{*}{ rs699947 } & Almawi & 382 & 393 & 135 & 183 & 64 & 165 & 178 & 50 & 0.48 \\
\hline & Bao & 55 & 52 & 34 & 5 & 16 & 26 & 09 & 17 & 0.17 \\
\hline & Ben Salem & 118 & 150 & 35 & 63 & 20 & 45 & 77 & 28 & 0.62 \\
\hline & Gomes & 87 & 84 & 27 & 38 & 22 & 18 & 41 & 25 & 0.70 \\
\hline & Vural & 137 & 155 & 52 & 63 & 22 & 52 & 78 & 25 & 0.64 \\
\hline \multirow[t]{2}{*}{ SNP } & Author & \multicolumn{2}{|c|}{ Sample size } & \multicolumn{3}{|c|}{ Case Genotype } & Cont & notyp & & HWE \\
\hline & & Case & Control & $A A$ & AG & GG & $A A$ & AG & GG & $\mathrm{Y} / \mathrm{N}(p)$ \\
\hline rs 1570360 & Almawi & 382 & 393 & 197 & 140 & 45 & 218 & 131 & 44 & 0.09 \\
\hline & Bao & 55 & 52 & 24 & 13 & 18 & 30 & 9 & 13 & 0.03 \\
\hline & Ben Salem & 118 & 150 & 57 & 42 & 19 & 75 & 57 & 18 & 0.17 \\
\hline & Gomes & 87 & 84 & 56 & 24 & 7 & 52 & 31 & 1 & 0.12 \\
\hline & $\mathrm{Li}$ & 110 & 100 & 78 & 29 & 3 & 65 & 30 & 5 & 0.53 \\
\hline
\end{tabular}




\section{Results}

\section{Study selection and characteristics}

A total of 129 articles were obtained through initial literature retrieval. After excluding 25 duplicates, the remaining 104 papers were filtrated by reading their titles and abstracts. After further screening, totally 12 papers entered the full-text acquisition and detailed screening. After excluding a repetitive study based on the same case group and another article focusing on the association between VEGF gene polymorphisms and insulin resistance in patients with PCOS, 10 articles met the inclusion criteria and eventually merged into the study. A total of 1347 PCOS cases and 1378 controls were included in the 10 included studies [20-29]. This analysis spanned seven regions and two ethnic groups, Caucasian and Asian. In all the incorporated studies, the diagnostic criteria of PCOS patients in the case group were Rotterdam criteria, and the inclusion criteria of the control group were clearly explained. The NOS score of all the included studies was higher than 6 points, and most of them explored the relationship between multiple SNPs and PCOS susceptibility. Therefore, in the final pooled analysis, 6 studies were eligible for VEGF gene rs2010963 polymorphism and PCOS risk (952 cases and 960 controls), 6 for rs 833061 polymorphism and PCOS risk (936 cases and 1010 controls), 5 for rs3025039 polymorphism and PCOS risk (769 cases and 779 controls), 5 for rs699947 polymorphism and PCOS risk (779 cases and 834controls), and 5 for rs1570360 polymorphism and PCOS risk (752 cases and 779 controls).

The flowchart of selection and inclusion process is displayed in Fig. 1, and the general characteristics of all included studies in Table 1. Table 2 shows the detailed genotype distributions of each group and the $p$ value of Hardy-Weinberg equilibrium (HWE) in each control group. The Newcastle-Ottawa Scale scores for all the included studies are shown in Table 3.

\section{Meta-analysis results}

Meta-analysis of association between VEGF gene rs2010963 polymorphism and PCOS susceptibility

The main results of the overall and subgroup metaanalysis for VEGF gene polymorphisms and PCOS susceptibility are presented in Table 4. Six studies had 952 cases and 960 controls for VEGF rs2010963 polymorphism. Four of them were based on the Asian populations and two on the Caucasian populations. Overall, the pooled results based on the whole population revealed significant correlation between VEGF rs2010963 variation and decreased PCOS susceptibility under 2 gene comparison models (Dominant model: $\mathrm{OR}=0.78$, 95\%CI: $0.65-0.94, P=0.008, \mathrm{I}^{2}=49 \%$; Heterozygote model: $\mathrm{OR}=0.75,95 \% \mathrm{CI}$ : 0.61-0.91, $P=0.04, \mathrm{I}^{2}=48 \%$ ). When the studies were stratified by ethnicity, significantly decreased risk of PCOS was observed in the Asian populations under 3 gene comparison models (Allele model: $\mathrm{OR}=0.76,95 \% \mathrm{CI}$ : $0.63-0.93, \mathrm{P}=0.008, \mathrm{I}^{2}=39 \%$;

Table 3 Quality assessment using the Newcastle-Ottawa scale

\begin{tabular}{|c|c|c|c|c|c|c|c|c|c|}
\hline Study & $\begin{array}{l}\text { Adequacy of } \\
\text { Case Definition }\end{array}$ & $\begin{array}{l}\text { Representativeness } \\
\text { of the Cases }\end{array}$ & $\begin{array}{l}\text { Selection of } \\
\text { Controls }\end{array}$ & $\begin{array}{l}\text { Definition of } \\
\text { Controls }\end{array}$ & $\begin{array}{l}\text { Compara- } \\
\text { bility }\end{array}$ & $\begin{array}{l}\text { Ascertainment } \\
\text { of exposure }\end{array}$ & $\begin{array}{l}\text { Same method of } \\
\text { ascertainment }\end{array}$ & $\begin{array}{l}\text { Non- } \\
\text { Response } \\
\text { rate }\end{array}$ & score \\
\hline $\begin{array}{l}\text { Almawi } \\
2016 \text { [20] }\end{array}$ & * & * & * & * & * & * & * & * & 8 \\
\hline $\begin{array}{l}\text { Bao } 2019 \\
{[21]}\end{array}$ & * & & * & * & & $*$ & $*$ & * & 6 \\
\hline $\begin{array}{l}\text { Ben Salem } \\
2016 \text { [22] }\end{array}$ & * & * & $*$ & * & & * & * & * & 7 \\
\hline $\begin{array}{l}\text { Ding } 2009 \\
\text { [28] }\end{array}$ & * & & * & * & & $*$ & * & * & 6 \\
\hline $\begin{array}{l}\text { Gomes } \\
2019 \text { [23] }\end{array}$ & * & & * & * & & $*$ & * & * & 6 \\
\hline $\begin{array}{l}\text { Guruvaiah } \\
2014[24]\end{array}$ & * & * & * & * & & * & * & * & 7 \\
\hline $\begin{array}{l}\text { Huang } \\
2019[25]\end{array}$ & * & * & * & * & & * & $*$ & * & 7 \\
\hline $\begin{array}{l}\text { Lee } 2008 \\
{[26]}\end{array}$ & * & * & & * & & $*$ & * & * & 6 \\
\hline Li 2014 [29] & * & * & & * & & $*$ & * & * & 6 \\
\hline $\begin{array}{l}\text { Vural } 2009 \\
\text { [27] }\end{array}$ & * & * & & * & & $*$ & * & * & 6 \\
\hline
\end{tabular}


Table 4 Main results of overall and subgroup analyses for VEGF polymorphisms and PCOS

\begin{tabular}{|c|c|c|c|c|c|c|c|c|c|c|c|c|c|c|c|c|}
\hline \multirow{2}{*}{$\begin{array}{l}\text { Group/ } \\
\text { Subgroup }\end{array}$} & \multirow{2}{*}{$\begin{array}{l}\text { Sample } \\
\text { size } \\
\text { (Case/ } \\
\text { Control) }\end{array}$} & \multicolumn{3}{|c|}{ Allele model } & \multicolumn{3}{|c|}{ Dominant model } & \multicolumn{3}{|c|}{ Heterozygote model } & \multicolumn{3}{|c|}{ Recessive model } & \multicolumn{3}{|c|}{ Homozygote model } \\
\hline & & $\begin{array}{l}\mathrm{OR} \\
(95 \% \mathrm{Cl})\end{array}$ & $p$ & $1^{2}$ & $\begin{array}{l}\mathrm{OR} \\
(95 \% \mathrm{Cl})\end{array}$ & $p$ & $1^{2}$ & $\begin{array}{l}\mathrm{OR} \\
(95 \% \mathrm{Cl})\end{array}$ & $p$ & $1^{2}$ & $\begin{array}{l}\mathrm{OR} \\
(95 \% \mathrm{Cl})\end{array}$ & $p$ & $1^{2}$ & $\begin{array}{l}\mathrm{OR} \\
(95 \% \mathrm{Cl})\end{array}$ & $p$ & $1^{2}$ \\
\hline \multicolumn{17}{|l|}{ rs2010963 } \\
\hline Total & $952 / 960$ & $\begin{array}{l}0.88 \text { [0.76, } \\
1.01]\end{array}$ & 0.06 & $48 \%$ & $\begin{array}{l}0.78[0.65, \\
0.94]\end{array}$ & 0.008 & $49 \%$ & $\begin{array}{l}0.75[0.61, \\
0.91]\end{array}$ & 0.004 & $48 \%$ & $\begin{array}{l}1.03[0.79, \\
1.36]\end{array}$ & 0.82 & $24 \%$ & $\begin{array}{l}0.88[0.65 \\
1.17]\end{array}$ & 0.38 & $28 \%$ \\
\hline Caucasian & $519 / 548$ & $\begin{array}{l}0.99[0.82, \\
1.20]\end{array}$ & 0.94 & $24 \%$ & $\begin{array}{l}0.98[0.55 \\
1.73]\end{array}$ & 0.93 & $75 \%$ & $\begin{array}{l}0.93[0.44 \\
1.96]\end{array}$ & 0.85 & $84 \%$ & $\begin{array}{l}1.41[0.93 \\
2.12]\end{array}$ & 0.10 & $0 \%$ & $\begin{array}{l}1.17[0.76, \\
1.80]\end{array}$ & 0.48 & $0 \%$ \\
\hline Asian & $433 / 412$ & $\begin{array}{l}0.76[0.63 \\
0.93]\end{array}$ & 0.008 & $39 \%$ & $\begin{array}{l}0.69[0.50, \\
0.95]\end{array}$ & 0.02 & $26 \%$ & $\begin{array}{l}0.68[0.50, \\
0.92]\end{array}$ & 0.01 & $0 \%$ & $\begin{array}{l}0.80[0.55 \\
1.16]\end{array}$ & 0.24 & $0 \%$ & $\begin{array}{l}0.68[0.46, \\
1.02]\end{array}$ & 0.06 & $21 \%$ \\
\hline PB & $834 / 830$ & $\begin{array}{l}0.92[0.79 \\
1.06]\end{array}$ & 0.24 & $45 \%$ & $\begin{array}{l}0.82[0.68, \\
1.00]\end{array}$ & 0.29 & $48 \%$ & $\begin{array}{l}0.83[0.59, \\
1.16]\end{array}$ & 0.27 & $51 \%$ & $\begin{array}{l}1.09[0.81, \\
1.47]\end{array}$ & 0.56 & $29 \%$ & $\begin{array}{l}0.95[0.69 \\
1.30]\end{array}$ & 0.73 & $24 \%$ \\
\hline \multicolumn{17}{|l|}{ rs833061 } \\
\hline Total & $\begin{array}{l}936 / \\
1010\end{array}$ & $\begin{array}{l}0.98[0.86, \\
1.12]\end{array}$ & 0.80 & $25 \%$ & $\begin{array}{l}0.93[0.77, \\
1.12]\end{array}$ & 0.45 & $6 \%$ & $\begin{array}{l}0.91[0.74, \\
1.11]\end{array}$ & 0.35 & $0 \%$ & $\begin{array}{l}1.06[0.84, \\
1.33]\end{array}$ & 0.63 & $0 \%$ & $\begin{array}{l}0.97[0.75 \\
1.26]\end{array}$ & 0.83 & $0 \%$ \\
\hline Caucasian & $637 / 698$ & $\begin{array}{l}0.99[0.84 \\
1.15]\end{array}$ & 0.85 & $22 \%$ & $\begin{array}{l}0.93[0.74, \\
1.16]\end{array}$ & 0.51 & $0 \%$ & $\begin{array}{l}0.90[0.71 \\
1.15]\end{array}$ & 0.4 & $0 \%$ & $\begin{array}{l}1.06[0.81, \\
1.40]\end{array}$ & 0.66 & $27 \%$ & $\begin{array}{l}1.00[0.73 \\
1.35]\end{array}$ & 0.98 & $28 \%$ \\
\hline Asian & $299 / 312$ & $\begin{array}{l}0.96[0.68 \\
1.37]\end{array}$ & 0.83 & $51 \%$ & $\begin{array}{l}0.90[0.54, \\
1.49]\end{array}$ & 0.67 & $54 \%$ & $\begin{array}{l}0.93[0.64, \\
1.33]\end{array}$ & 0.68 & $47 \%$ & $\begin{array}{l}1.05[0.68, \\
1.62]\end{array}$ & 0.84 & $0 \%$ & $\begin{array}{l}0.92[0.57 \\
1.48]\end{array}$ & 0.73 & $0 \%$ \\
\hline PB & $818 / 880$ & $\begin{array}{l}0.95[0.83, \\
1.09]\end{array}$ & 0.48 & $10 \%$ & $\begin{array}{l}0.87[0.71, \\
1.07]\end{array}$ & 0.18 & $0 \%$ & $\begin{array}{l}0.85[0.68, \\
1.06]\end{array}$ & 0.14 & $0 \%$ & $\begin{array}{l}1.04[0.82, \\
1.32]\end{array}$ & 0.74 & $0 \%$ & $\begin{array}{l}0.94[0.72, \\
1.23]\end{array}$ & 0.63 & $0 \%$ \\
\hline \multicolumn{17}{|l|}{ rs3025039 } \\
\hline Total & $779 / 834$ & $\begin{array}{l}0.97[0.68, \\
1.39]\end{array}$ & 0.88 & $65 \%$ & $\begin{array}{l}1.07[0.73, \\
1.57]\end{array}$ & 0.73 & $58 \%$ & $\begin{array}{l}1.21[0.95 \\
1.55]\end{array}$ & 0.12 & $42 \%$ & $\begin{array}{l}0.59[0.34, \\
1.02]\end{array}$ & 0.06 & $0 \%$ & $\begin{array}{l}0.56[0.32, \\
0.99]\end{array}$ & 0.05 & $0 \%$ \\
\hline Caucasian & $500 / 543$ & $\begin{array}{l}1.22[0.93 \\
1.60]\end{array}$ & 0.16 & $0 \%$ & $\begin{array}{l}1.35[1.00, \\
1.83]\end{array}$ & 0.05 & $10 \%$ & $\begin{array}{l}1.43[1.04 \\
1.95]\end{array}$ & 0.03 & $33 \%$ & $\begin{array}{l}0.70[0.27, \\
1.82]\end{array}$ & 0.46 & $0 \%$ & $\begin{array}{l}0.76[0.29 \\
1.97]\end{array}$ & 0.57 & $0 \%$ \\
\hline Asian & $269 / 236$ & $\begin{array}{l}0.79[0.48, \\
1.29]\end{array}$ & 0.35 & $63 \%$ & $\begin{array}{l}0.83[0.49, \\
1.41]\end{array}$ & 0.49 & $52 \%$ & $\begin{array}{l}0.94[0.63 \\
1.39]\end{array}$ & 0.75 & $26 \%$ & $\begin{array}{l}0.54[0.28 \\
1.05]\end{array}$ & 0.07 & $48 \%$ & $\begin{array}{l}0.48[0.24 \\
0.97]\end{array}$ & 0.04 & $36 \%$ \\
\hline PB & 689/695 & $\begin{array}{l}0.91[0.60 \\
1.39]\end{array}$ & 0.68 & $71 \%$ & $\begin{array}{l}1.00[0.63, \\
1.60]\end{array}$ & 0.99 & $66 \%$ & $\begin{array}{l}1.13[0.72, \\
1.76]\end{array}$ & 0.60 & $55 \%$ & $\begin{array}{l}0.59[0.34, \\
1.02]\end{array}$ & 0.06 & $0 \%$ & $\begin{array}{l}0.56[0.32, \\
0.99]\end{array}$ & 0.05 & $0 \%$ \\
\hline $\begin{array}{l}\text { HWE } \\
\text { (yes) }\end{array}$ & $571 / 545$ & $\begin{array}{l}0.79 \text { [0.50, } \\
1.25]\end{array}$ & 0.32 & $70 \%$ & $\begin{array}{l}0.84[0.51 \\
1.40]\end{array}$ & 0.51 & $63 \%$ & $\begin{array}{l}1.06[0.79, \\
1.41]\end{array}$ & 0.71 & $41 \%$ & $\begin{array}{l}0.58[0.33, \\
1.04]\end{array}$ & 0.07 & $4 \%$ & $\begin{array}{l}0.55[0.30, \\
0.99]\end{array}$ & 0.05 & $0 \%$ \\
\hline \multicolumn{17}{|l|}{ rs699947 } \\
\hline Total & 779/834 & $\begin{array}{l}1.03[0.90, \\
1.19]\end{array}$ & 0.65 & $49 \%$ & $\begin{array}{l}1.03[0.84, \\
1.26]\end{array}$ & 0.78 & $48 \%$ & $\begin{array}{l}1.01 \text { [0.81, } \\
1.26]\end{array}$ & 0.91 & $37 \%$ & $\begin{array}{l}1.07[0.82, \\
1.38]\end{array}$ & 0.62 & $0 \%$ & $\begin{array}{l}1.06 \text { [0.80, } \\
1.41]\end{array}$ & 0.69 & $36 \%$ \\
\hline Caucasian & $724 / 782$ & $\begin{array}{l}1.00[0.80 \\
1.25]\end{array}$ & 0.99 & $51 \%$ & $\begin{array}{l}1.07[0.87, \\
1.32]\end{array}$ & 0.53 & $49 \%$ & $\begin{array}{l}1.05[0.83, \\
1.31]\end{array}$ & 0.70 & $30 \%$ & $\begin{array}{l}1.09[0.83, \\
1.43]\end{array}$ & 0.52 & $0 \%$ & $\begin{array}{l}1.11[0.82, \\
1.51]\end{array}$ & 0.49 & $44 \%$ \\
\hline \multicolumn{17}{|l|}{ rs1570360 } \\
\hline Total & $752 / 779$ & $\begin{array}{l}1.11[0.95, \\
1.30]\end{array}$ & 0.20 & $0 \%$ & $\begin{array}{l}1.15[0.94, \\
1.41]\end{array}$ & 0.17 & $11 \%$ & $\begin{array}{l}0.69[0.37, \\
1.27]\end{array}$ & 0.23 & $82 \%$ & $\begin{array}{l}1.23[0.89, \\
1.70]\end{array}$ & 0.21 & $15 \%$ & $\begin{array}{l}1.29[0.92, \\
1.80]\end{array}$ & 0.14 & $13 \%$ \\
\hline Caucasian & $587 / 627$ & $\begin{array}{l}1.12[0.94 \\
1.34]\end{array}$ & 0.19 & $0 \%$ & $\begin{array}{l}1.18 \text { [0.94, } \\
1.49]\end{array}$ & 0.14 & $0 \%$ & $\begin{array}{l}0.58[0.24 \\
1.37]\end{array}$ & 0.21 & $88 \%$ & $\begin{array}{l}1.26[0.88, \\
1.81]\end{array}$ & 0.21 & $40 \%$ & $\begin{array}{l}1.31[0.90 \\
1.90]\end{array}$ & 0.16 & $23 \%$ \\
\hline Asian & $165 / 152$ & $\begin{array}{l}1.08[0.53 \\
2.23]\end{array}$ & 0.83 & $73 \%$ & $\begin{array}{l}1.11[0.49, \\
2.53]\end{array}$ & 0.79 & $66 \%$ & $\begin{array}{l}0.95[0.24 \\
3.81]\end{array}$ & 0.95 & $82 \%$ & $\begin{array}{l}1.12[0.55 \\
2.30]\end{array}$ & 0.75 & $27 \%$ & $\begin{array}{l}1.22[0.58 \\
2.57]\end{array}$ & 0.60 & $50 \%$ \\
\hline PB & $642 / 679$ & $\begin{array}{l}1.16[0.98, \\
1.37]\end{array}$ & 0.08 & $0 \%$ & $\begin{array}{l}1.22[0.98, \\
1.52]\end{array}$ & 0.07 & $0 \%$ & $\begin{array}{l}0.75[0.35 \\
1.58]\end{array}$ & 0.44 & $84 \%$ & $\begin{array}{l}1.29[0.92, \\
1.79]\end{array}$ & 0.14 & $13 \%$ & $\begin{array}{l}1.36[0.96 \\
1.93]\end{array}$ & 0.08 & $0 \%$ \\
\hline $\begin{array}{l}\text { HWE } \\
\text { (yes) }\end{array}$ & $697 / 727$ & $\begin{array}{l}1.08 \text { [0.91, } \\
1.27]\end{array}$ & 0.39 & $0 \%$ & $\begin{array}{l}1.12[0.90, \\
1.38]\end{array}$ & 0.30 & $8 \%$ & $\begin{array}{l}0.56[0.29 \\
1.09]\end{array}$ & 0.09 & $84 \%$ & $\begin{array}{l}1.19[0.84, \\
1.69]\end{array}$ & 0.32 & $33 \%$ & $\begin{array}{l}1.23[0.85 \\
1.76]\end{array}$ & 0.27 & $25 \%$ \\
\hline
\end{tabular}

VEGF vascular endothelial growth factor, $P C O S$ polycystic ovary syndrome, $O R$ odds ratio, $C l$ confidence interval, $N A$ not available, $H B$ hospital-based, $P B$ population-based. The bold values represent statistically significant differences

Dominant model: $\mathrm{OR}=0.69,95 \% \mathrm{CI}: 0.50-0.95, P=0.02$, $\mathrm{I}^{2}=26 \%$; Heterozygote model: $\mathrm{OR}=0.68,95 \% \mathrm{CI}: 0.50$ $0.92, P=0.01, \mathrm{I}^{2}=0 \%$. No significant association was found between VEGF rs2010963 polymorphism and PCOS susceptibility in the Caucasian ethnicities and stratified subgroup analyses based on source of control. 


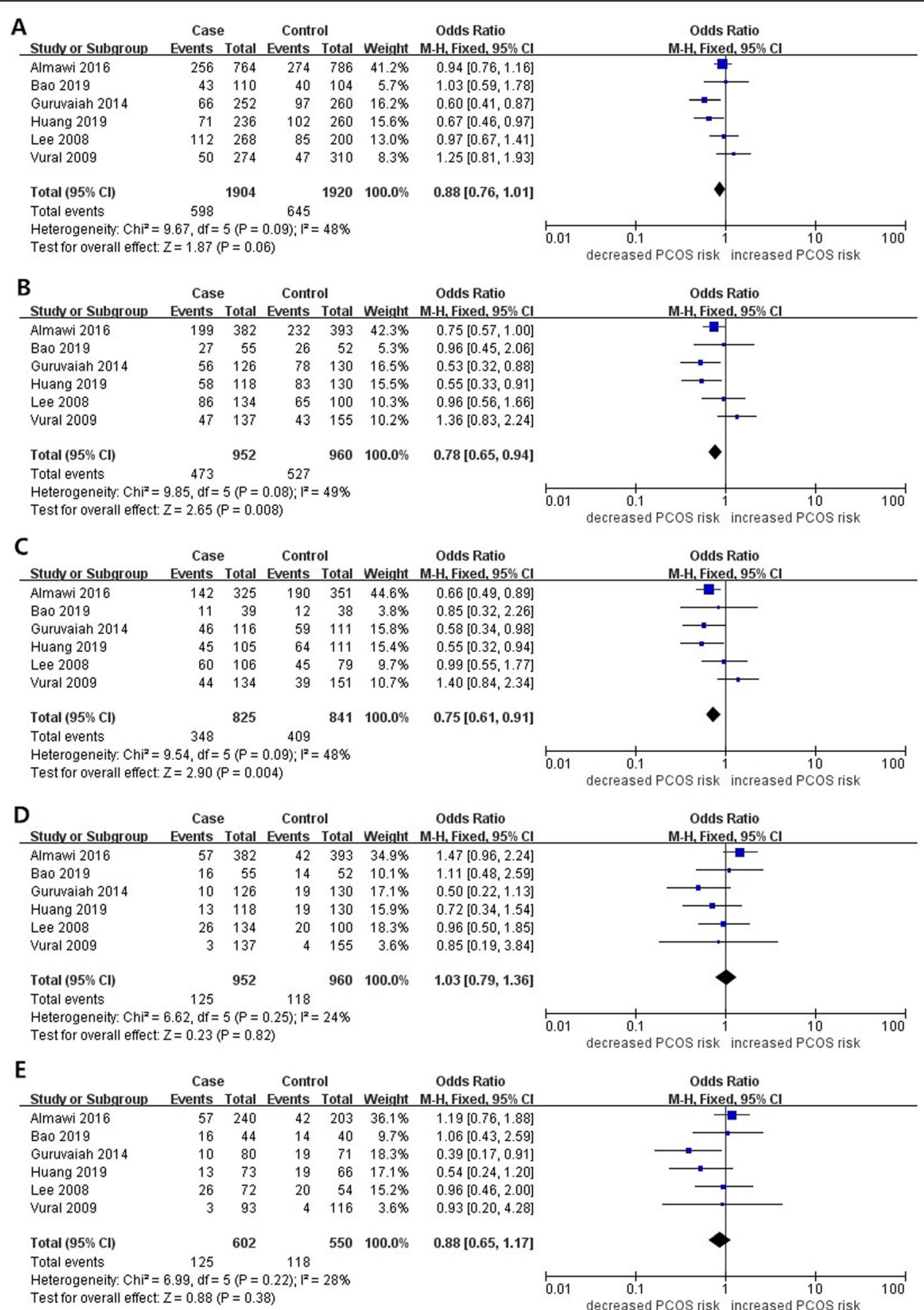

Fig. 2 Forest plots for association between VEGF gene rs2010963 polymorphism and PCOS susceptibility in different genetic models (a: allele model $\mathbf{b}$ :dominant model cheterozygous model $\mathbf{d}$ :recessive model e:homozygous model)

Figure 2 shows the forest plot of the association between VEGF gene rs2010963 polymorphism and PCOS susceptibility.

\section{Meta-analysis of association between VEGF gene rs833061 polymorphism and PCOS susceptibility}

Six studies had 936 cases and 1010 controls for VEGF rs2010963 polymorphism, 3 of which were based on the Asian populations and another 3 on the Caucasian populations. There was no significant correlation found between
VEGF gene rs833061 polymorphism and risk of PCOS, whether based on aggregate analysis of all populations or subgroup analyses based on different ethnicities and sources of control.

\section{Meta-analysis of association between VEGF gene rs3025039 polymorphism and PCOS susceptibility}

Five studies had 769 cases and 779 controls for VEGF rs3025039 polymorphism, 3 of which were based on the Asian populations and 2 on the Caucasian populations. 
Overall, the pooled results based on the whole population revealed significantly negative correlation between VEGF rs2010963 polymorphism and PCOS risk under Homozygote model $\left(\mathrm{OR}=0.56,95 \% \mathrm{CI}: 0.32-0.99, P=0.05, \mathrm{I}^{2}=\right.$ $0 \%)$, Based on ethnic stratification, significantly increased risk of PCOS was observed in the Caucasian populations under 2 gene comparison models (Dominant model: OR = 1.35, 95\%CI: $1.00-0.95, \mathrm{P}=0.05, \mathrm{I}^{2}=10 \%$; Heterozygote model: $\mathrm{OR}=1.43,95 \% \mathrm{CI}: 1.04-1.95, P=0.03, \mathrm{I}^{2}=33 \%$ ). However, in the Asian populations, there was negative correlation between VEGF rs2010963 polymorphism and PCOS risk under Homozygote model $(\mathrm{OR}=0.48,95 \% \mathrm{CI}$ : 0.24-0.97, $P=0.04, \mathrm{I}^{2}=36 \%$ ). Moreover, subgroup analysis based on source of control indicated that VEGF rs3025039 polymorphism was related to decreased PCOS risk in the population-based group under Homozygote model $\left(\mathrm{OR}=0.56,95 \% \mathrm{CI}\right.$ : $\left.0.32-0.99, P=0.05, \mathrm{I}^{2}=0 \%\right)$.

\section{Meta-analysis of association between VEGF gene rs699947} polymorphism and PCOS susceptibility

Five studies had 779 cases and 834 controls for VEGF rs699947 polymorphism. Four of them were based on the Caucasian populations. There was no significant correlation between VEGF gene rs699947 polymorphism and risk of PCOS, whether based on analysis by synthesis of all populations or subgroup analyses based on the Caucasian ethnicities.

\section{Meta-analysis of association between VEGF gene rs1570360polymorphism and PCOS susceptibility}

Five studies had 752 cases and 779 controls for VEGF rs1570360 polymorphism. Three of them were based on the Caucasian populations and two on the Asian populations. No significant association was identified between VEGF gene rs1570360 polymorphism and risk of PCOS, whether based on aggregate analysis of all populations or subgroup analyses based on different ethnicities and sources of control.

\section{Publication bias}

Statistical association was found between VEGF rs2010963 and rs3025039 polymorphisms and PCOS susceptibility. The potential publication bias was first evaluated by observing the funnel plot obtained through Revman5.3 software. Funnel plots showed no conspicuous visual asymmetry (Fig. 3). Begg's test and Egger's test were applied to further detect potential publication bias. The results of Begg's test and Egger's test also revealed no significant evidence of publication bias. Table 5 summarizes the main results of Begg's test and Egger's test.

\section{Sensitivity analysis}

Sensitivity analysis was performed to assess the influence of each individual study on the overall pooled results. By sequentially removing individual study, no statistical variation of pooled OR was observed. The results of sensitivity analysis indicated that the pooled ORs of this meta-analysis were stable (Fig. 4).

\section{Trial sequential analysis}

Trial sequential analysis was performed for each gene comparison model with statistically significant differences obtained through previous calculation to investigate whether the pooled results we obtained were stable

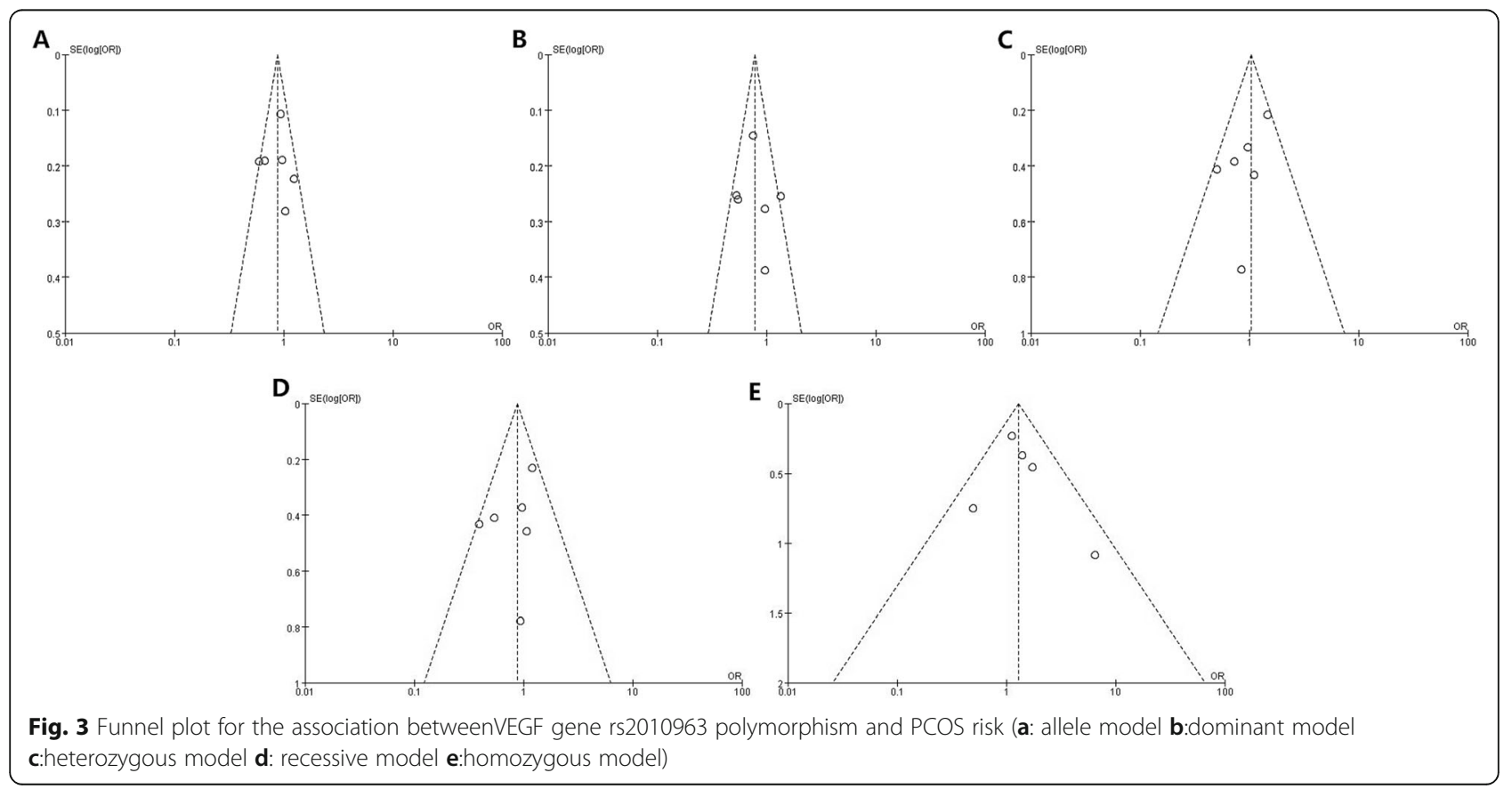


Table 5 Summary of publication bias tests

\begin{tabular}{|c|c|c|c|c|c|}
\hline \multirow[t]{2}{*}{ SNP } & \multirow{2}{*}{$\begin{array}{l}\text { Comparison model } \\
\text { type }\end{array}$} & \multicolumn{2}{|c|}{ begg's text } & \multicolumn{2}{|c|}{ egger's text } \\
\hline & & z & $\boldsymbol{p}$ value & $\mathrm{t}$ & $\boldsymbol{p}$ value \\
\hline \multirow[t]{5}{*}{ rs2010963 } & Allele model & -0.19 & 0.851 & 0.04 & 0.969 \\
\hline & Dominant model & 0.56 & 0.573 & 0.48 & 0.655 \\
\hline & Heterozygote model & 0.56 & 0.576 & 1.31 & 0.261 \\
\hline & Recessive model & -0.56 & 0.573 & -2.01 & 0.115 \\
\hline & Homozygote model & -0.94 & 0.348 & -1.31 & 0.260 \\
\hline \multirow[t]{5}{*}{ rs833061 } & Allele model & 0.19 & 0.851 & -0.50 & 0.641 \\
\hline & Dominant model & -0.94 & 0.348 & 3.15 & 0.035 \\
\hline & Heterozygote model & -1.32 & 0.188 & -0.45 & 0.679 \\
\hline & Recessive model & -0.19 & 0.851 & -0.55 & 0.608 \\
\hline & Homozygote model & -0.19 & 0.851 & -0.33 & 0.760 \\
\hline \multirow[t]{5}{*}{ rs3025039 } & Allele model & 0.49 & 0.624 & -0.01 & 0.993 \\
\hline & Dominant model & 0.00 & 1.000 & -0.22 & 0.837 \\
\hline & Heterozygote model & 0.49 & 0.624 & -0.23 & 0.835 \\
\hline & Recessive model & -1.36 & 0.174 & -0.66 & 0.576 \\
\hline & Homozygote model & -0.68 & 0.497 & 1.17 & 0.362 \\
\hline \multirow[t]{5}{*}{ rs699947 } & Allele model & -1.47 & 0.142 & -5.82 & 0.010 \\
\hline & Dominant model & -1.47 & 0.142 & -5.19 & 0.014 \\
\hline & Heterozygote model & -0.98 & 0.327 & -3.59 & 0.037 \\
\hline & Recessive model & -1.47 & 0.142 & -5.07 & 0.015 \\
\hline & Homozygote model & -1.47 & 0.142 & -10.90 & 0.002 \\
\hline \multirow[t]{5}{*}{ rs1570360 } & Allele model & 0.98 & 0.327 & 0.24 & 0.828 \\
\hline & Dominant model & 0.00 & 1.000 & -0.43 & 0.696 \\
\hline & Heterozygote model & -0.49 & 0.624 & -0.50 & 0.651 \\
\hline & Recessive model & 1.47 & 0.142 & 1.49 & 0.233 \\
\hline & Homozygote model & 1.47 & 0.142 & 1.43 & 0.249 \\
\hline
\end{tabular}

and reliable. Under the dominant model of rs2010963, although the cumulative $\mathrm{Z}$ curve did not intersect with the trial sequential monitoring boundary, the cumulative case samples exceeded the required information size (RIS), suggesting that the accumulated sample size was sufficient and the pooled positive result was credible. Under the heterozygote model of rs2010963, the cumulative $\mathrm{Z}$ curve passed through the traditional boundary threshold, meanwhile crossed the trial sequential monitoring boundary, and confirmed a reliable conclusion although the cumulative sample size did not transcend the required information size (RIS). In other gene comparison models that yielded positive results, the cumulative $\mathrm{Z}$ curve did not cross the sequential trial monitoring boundary, and the sample size did not reach RIS, demonstrating that the pooled results possibly were not solid enough. Figure 5 shows the results of trial sequential analysis under the dominant model and the heterozygote model of rs2010963.

\section{Discussion}

Polycystic ovary syndrome (PCOS) is a female endocrine disorder with complex etiology. Currently, the widely adopted diagnostic criteria are Rotterdam criteria: rare ovulation or anovulation, clinical or biochemical hyperandrogenemia, and ovarian polycystic changes determined by ultrasound. Patients with two of three in the criteria and without other diseases that may cause hyperandrogenemia could be diagnosed with PCOS [30]. The prominent pathophysiological features of PCOS are hyperplasia and hypervascularity of the ovarian theca interna and stroma [2]. Angiogenesis in the ovary is essential for follicular growth, ovulation, and subsequent luteal development and regression. Increasing evidence demonstrates that there are multiple dysregulation of angiogenic factors in PCOS, including vascular endothelial growth factor, angiogenin, platelet-derived growth factor, transforming growth factor-alpha, and basic fibroblast growth factor. VEGF is one of the major regulators of angiogenesis, and its important role in physiological and pathological angiogenesis has been proved. It is the most extensively studied angiogenic factor related to the pathophysiology of PCOS [35]. In the female reproductive system, macrophages and granulosa cells are the main sources of VEGF production. As a homologous dimeric glycoprotein hormone that binds heparin, VEGF can effectively promote vascular formation and improve vascular permeability [36]. VEGF protein family consists of 6 members, namely VEGF (also known as VEGFA), VEGFB, VEGFC, VEGFD, VEGFE and placenta growth factor (PLGF), with molecular weight ranging from 35 $\mathrm{kDa}$ to $45 \mathrm{kDa}$. VEGF is recognized as a "central regulator" of angiogenesis due to its ability to specifically promote vascular endothelial division, proliferation and migration, and is the most potent angiogenic factor [3639].

VEGF protein is known to be closely related to the pathogenesis of PCOS. Serum VEGF levels are significantly elevated in PCOS patients [16-18]. High levels of serum VEGF result in a higher number of active granuloas lutein cells (GLCs), increase secretion of GLCs, and up-regulate VEGF gene expression [40]. In addition, the expression of VEGF in the stromal hyperplasia of PCOS is significantly increased, resulting in an increase in microvascular permeability, and a large number of neovascularization may lead to dysplasia of the ovarian producing androgen. VEGF gene is located at $6 \mathrm{p} 21.3$, with a total length of about $14 \mathrm{~KB}$, and is alternately composed of 8 exons and 7 introns. There are at least 30 single nucleotide polymorphisms (SNPs) in the coding region of VEGF gene. Based on previous studies, rs2010963 and rs3025039 mutation sites have been proved to alter the plasma level of VEGF [41, 42]. The correlation between VEGF gene polymorphisms and the risk of PCOS has 

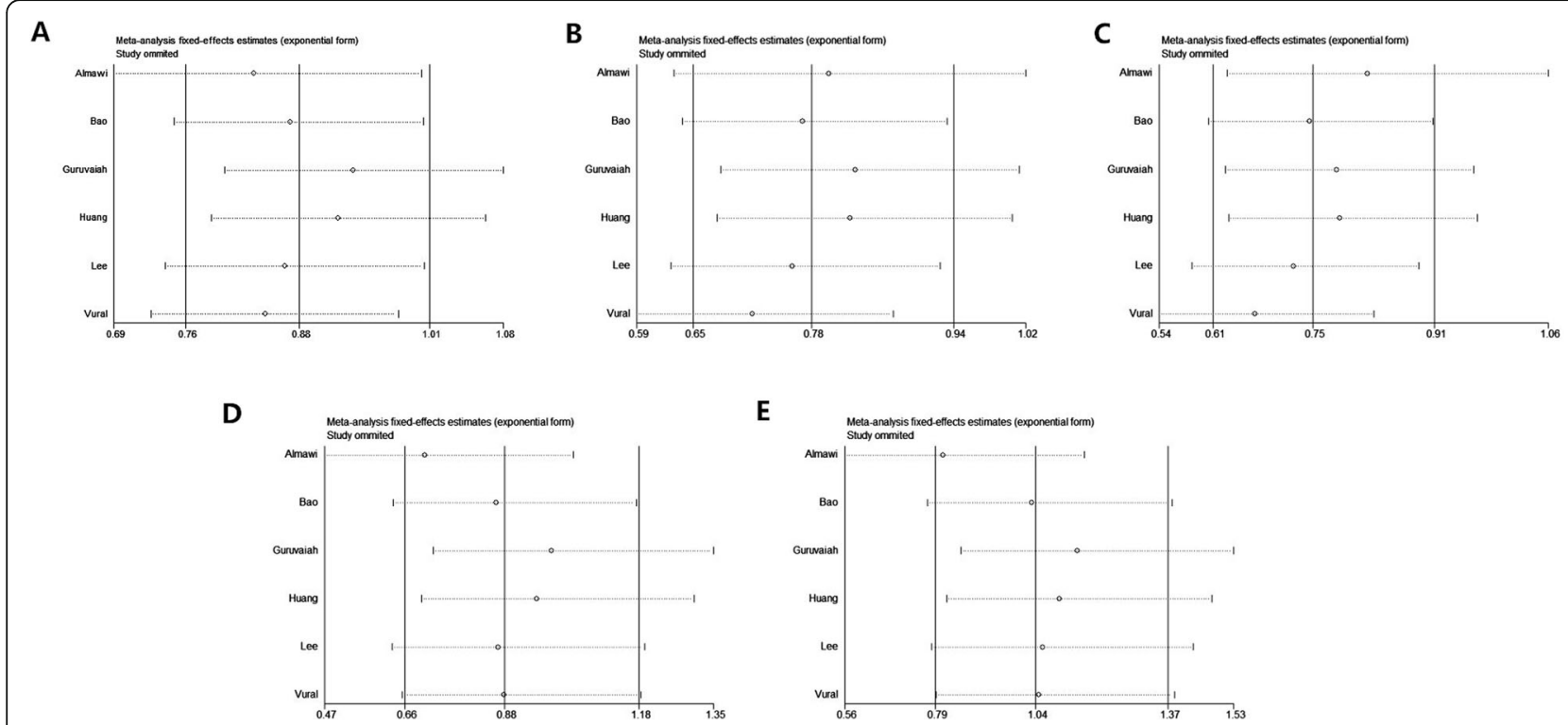

Fig. 4 Sensitivity analysis results between VEGF gene rs2010963 polymorphism and PCOS risk (a: allele model b:dominant model c:heterozygous model $\mathbf{d}$ : recessive model e:homozygous model)

attracted growing attention in recent years, among which the most widely investigated SNPs are rs2010963 $(\mathrm{G}>\mathrm{C}), \operatorname{rs} 833061(\mathrm{~T}>\mathrm{C}), \mathrm{rs} 3025039(\mathrm{C}>\mathrm{T}), \mathrm{rs} 699947$ $(\mathrm{A}>\mathrm{C})$, and $\operatorname{rs} 1570360(\mathrm{~A}>\mathrm{G})$, but the results from different studies remain highly controversial. For example, in studies on the correlation between rs2010963 and PCOS susceptibility, 3 studies reported no significant correlation between the two, while the other 3 studies showed a reduced risk of PCOS in C allele carriers. In the studies on the correlation between rs833061 and PCOS susceptibility, except that BAO found that there was a significant statistical significance between this SNP and PCOS susceptibility, all the other 5 studies revealed that it was not related to the risk of PCOS. Therefore, this meta-analysis was conducted to comprehensively evaluate the relationship between VEGF gene polymorphisms and PCOS risk based on the data in the existing literature. According to the NOS standard, all 10 incorporated studies were considered to have high-quality. In the present study, the results of the pooled analysis showed a significant correlation between VEGF rs2010963 variability and decreased susceptibility to PCOS. Subgroup analysis based on different ethnic stratification showed that the negative correlation between rs2010963 mutation and PCOS susceptibility only appeared in the Asian races, and there was no significant correlation in the Caucasian populations. The pooled analysis of the association between the VEGF gene rs3025039 polymorphism and the risk of PCOS showed that this SNP increased the risk of PCOS in the general
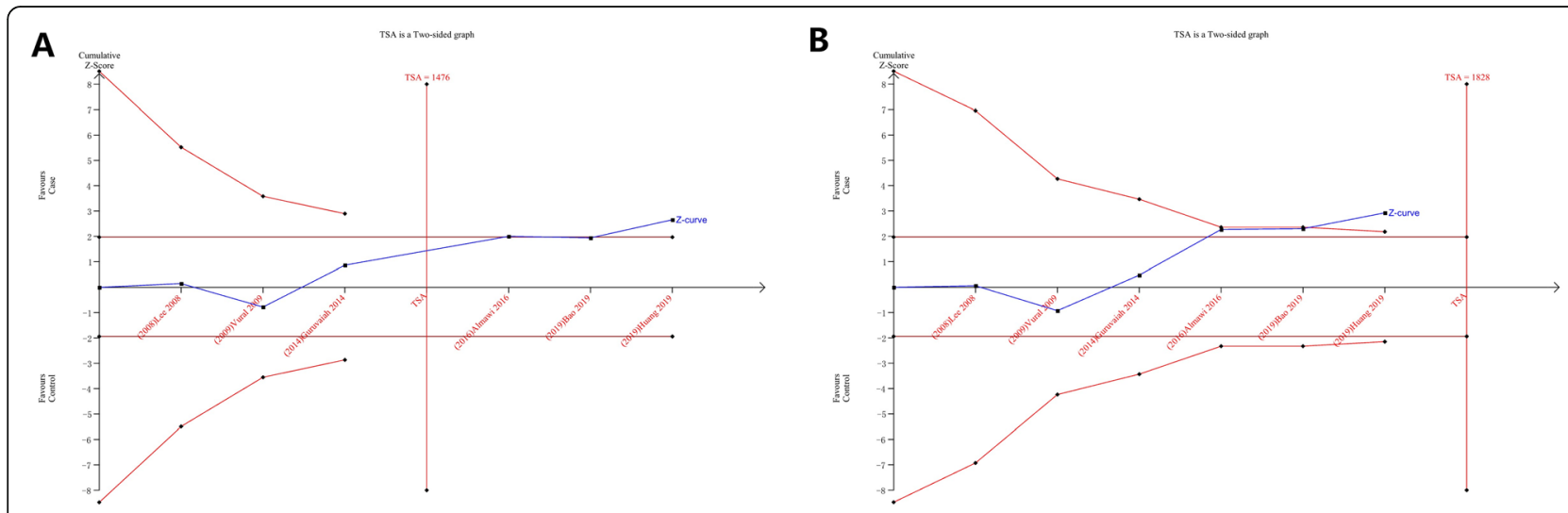

Fig. 5 Trial sequential analysis results of the association between VEGF gene rs2010963 polymorphism and PCOS risk (a:dominant model b: heterozygous model) 
population. Subgroup analysis based on different ethnicities showed that the rs3025039 polymorphism in the Caucasian populations was also associated with an increased risk of PCOS. In the Asian populations, the VEGF gene rs3025039 polymorphism reduced the risk of PCOS. Therefore, our results showed that the correlation between VEGF gene polymorphisms and the risk of PCOS in different populations may be quite different, suggesting that further experimental studies are needed to explore the possible underlying reasons for this difference.

It must be recognized that this meta-analysis has some possible limitations. First, the number of articles included in the study was small, and the overall number of patients in the case group and control group was low. Some subgroup analyses were unable to perform data analysis because only one study was included, thus limiting the reliability of summary results. Secondly, the previous studies on VEGF gene polymorphisms and PCOS risk only contained two ethnicities, lacking relevant studies on other races. Third, although the inclusion criteria for the control group were clearly stated in all studies, the inclusion criteria for different studies were not the same. For example, in some studies, the inclusion criteria for the control group were healthy women of childbearing age, while in others the criteria detailed a history of successful childbearing and a recent absence of any medications that might affect hormone levels. As PCOS is a disease with complex etiology, its incidence is related to many factors, such as obesity and insulin resistance. Due to the absence of relevant patient data based on the two factors in the original study literature, we did not conduct subgroup stratification analysis based on factors such as whether the patient was obese or not and the severity of insulin resistance.

Despite some limitations, this meta-analysis still has several highlights. To date, this is the first known metaanalysis focused on the correlation between VEGF gene polymorphisms and PCOS risk. Through the establishment of strict inclusion and exclusion criteria, a total of 10 studies were included in this meta-analysis, involving 1347 PCOS cases and 1378 controls. The study spanned seven regions and included both Caucasian and Asian populations. We systematically evaluated the effect of VEGF gene polymorphism on the occurrence of PCOS. At the same time, in most gene comparison models, the calculated inter-study heterogeneity was low, indicating the results of this meta-analysis were stable. In addition, the results of trial sequential analysis (TSA) also demonstrate the correlation between rs2010963 and decreased risk of PCOS obtained through this current metaanalysis was stable and reliable. Many previous studies have suggested that multiple SNPs of VEGF gene increase the risk of diseases such as ovarian malignancies, hypertensive disorder of pregnancy, and idiopathic recurrent pregnancy loss, and thereafter have been confirmed by multiple meta-analyses. Our meta-analysis results revealed certain VEGF gene polymorphisms had a distinct different impact on the pathogenesis of PCOS in contrast to its role in the pathogenesis of other female reproductive system disorders [43-45]. There was a significant correlation between VEGF rs2010963 and decreased PCOS susceptibility, suggesting VEGF rs2010963 could be a protective factor for the risk of PCOS.

In conclusion, this study establishes a significant correlation between the variation of VEGF rs2010963 and decreased PCOS susceptibility in the entire population; subgroup analysis shows this association only occurs occurred in Asian populations. The mutation of VEGF gene rs3025039 may also decrease the risk of PCOS in the overall population; subgroup analysis based on different populations shows that rs3025039 polymorphism is also associated with decreased risk of PCOS in the Asian populations, while in the Caucasian populations rs3025039 polymorphism of VEGF gene enhances the risk of PCOS. More well-designed studies involving more cases will be needed in the future to confirm this inference.

\section{Abbreviations \\ Cl: Confidence interval; HWE: Hardy-Weinberg equilibrium; OR: Odds ratio; PCOS: Polycystic ovarian syndrome; SNP: Single nucleotide polymorphism; VEGF: Vascular endothelial growth factor}

\section{Acknowledgements \\ None.}

\section{Authors' contributions}

$\mathrm{JZ}$ and LT conceived and designed the study. DL and JZ extracted the data. $\mathrm{DL}$ performed the statistical analysis. JZ wrote the first draft of the manuscript. All authors reviewed and edited the manuscript and approved the final version of the manuscript.

\section{Funding}

This work was supported by Jiangsu Basic Research Program (Natural

Science Foundation, BK20161298), Jiangsu Commission of Health (H2018014).

Availability of data and materials

The current study was based on results of relevant published studies.

Ethics approval and consent to participate

Not applicable.

Consent for publication

Not applicable.

Competing interests

The authors declare that they have no competing interests.

\section{Author details}

'Department of Reproductive Medicine, Lianyungang Maternal and Child Health Hospital, NO.669 Qindongmen Road, Lianyungang 222001, Jiangsu Province, China. ${ }^{2}$ Department of Vascular Surgery, the First People's Hospital of Lianyungang, NO.182 North Tongguan Road, Lianyungang, Jiangsu Province 222002, China. 
Received: 19 November 2019 Accepted: 24 February 2020 Published online: 09 March 2020

\section{References}

1. Li R, Zhang Q, Yang D, Li S, Lu S, Wu X, et al. Prevalence of polycystic ovary syndrome in women in China: a large community-based study. Hum Reprod. 2013;28(9):2562-9.

2. Hart R, Hickey M, Franks S. Definitions, prevalence and symptoms of polycystic ovaries and polycystic ovary syndrome. Best Pract Res Clin Obstetrics Gynaecol. 2004;18(5):671-83.

3. Witchel SF, Oberfield SE, Pena AS. Polycystic ovary syndrome: pathophysiology, presentation, and treatment with emphasis on adolescent girls. J Endocrine Soc. 2019;3(8):1545-73.

4. Catteau-Jonard S, Dewailly D. Pathophysiology of polycystic ovary syndrome: the role of hyperandrogenism. Front Horm Res. 2013;40:22-7.

5. Cunningham P. Pathophysiology, diagnosis and treatment of polycystic ovary syndrome. Nurs Stand. 2017;31(39):44-51.

6. Gorry A, White DM, Franks S. Infertility in polycystic ovary syndrome: focus on low-dose gonadotropin treatment. Endocrine. 2006;30(1):27-33.

7. Thessaloniki EA-SPCWG. Consensus on infertility treatment related to polycystic ovary syndrome. Fertil Steril. 2008;89(3):505-22.

8. Rai R, Backos M, Rushworth F, Regan L. Polycystic ovaries and recurrent miscarriage--a reappraisal. Hum Reprod. 2000;15(3):612-5

9. Chakraborty P, Goswami SK, Rajani S, Sharma S, Kabir SN, Chakravarty $B$, et al. Recurrent pregnancy loss in polycystic ovary syndrome: role of hyperhomocysteinemia and insulin resistance. PLoS One. 2013;8(5): e64446.

10. Azziz R. PCOS in 2015: new insights into the genetics of polycystic ovary syndrome. Nat Rev Endocrinol. 2016;12(2):74-5.

11. Mykhalchenko K, Lizneva D, Trofimova T, Walker W, Suturina L, Diamond MP, et al. Genetics of polycystic ovary syndrome. Expert Rev Mol Diagn. 2017;17(7):723-33.

12. Welt CK, Duran JM. Genetics of polycystic ovary syndrome. Semin Reprod Med. 2014;32(3):177-82.

13. Barber TM, Franks S. Genetics of polycystic ovary syndrome. Front Horm Res. 2013;40:28-39.

14. Ferrara N, Henzel WJ. Pituitary follicular cells secrete a novel heparin-binding growth factor specific for vascular endothelial cells. Biochem Biophys Res Commun. 1989;161(2):851-8.

15. Eng L, Azad AK, Habbous S, Pang V, Xu W, Maitland-van der Zee AH, et al. Vascular endothelial growth factor pathway polymorphisms as prognostic and pharmacogenetic factors in cancer: a systematic review and meta-analysis. Clin Cancer Res. 2012;18(17):4526-37.

16. Dambala K, Vavilis D, Bili E, Goulis DG, Tarlatzis BC. Serum visfatin, vascular endothelial growth factor and matrix metalloproteinase-9 in women with polycystic ovary syndrome. Gynecol Endocrinol. 2017;33(7):529-33.

17. Dambala K, Paschou SA, Michopoulos A, Siasos G, Goulis DG, Vavilis D, et al. Biomarkers of endothelial dysfunction in women with polycystic ovary syndrome. Angiology. 2019;70(9):797-801.

18. Agrawal R, Sladkevicius P, Engmann L, Conway GS, Payne NN, Bekis J, et al. Serum vascular endothelial growth factor concentrations and ovarian stromal blood flow are increased in women with polycystic ovaries. Hum Reprod. 1998;13(3):651-5.

19. Ferrara N, Frantz G, LeCouter J, Dillard-Telm L, Pham T, Draksharapu $A$, et al. Differential expression of the angiogenic factor genes vascular endothelial growth factor (VEGF) and endocrine glandderived VEGF in normal and polycystic human ovaries. Am J Pathol. 2003;162(6):1881-93.

20. Almawi WY, Gammoh E, Malalla ZH, Al-Madhi SA. Analysis of VEGFA variants and changes in VEGF levels underscores the contribution of VEGF to polycystic ovary syndrome. PloS one. 2016;11(11):e0165636.

21. Bao L, Syed R, Aloahd MS. Analysis of VEGF gene polymorphisms and serum VEGF protein levels contribution in polycystic ovary syndrome of patients. Mol Biol Rep. 2019;46(6):5821-9.

22. Ben, Salem A, Megdich F, Kacem O, Souayeh M, Hachani Ben Ali F, Hizem $S$, et al. Vascular endothelial growth factor (VEGFA) gene variation in polycystic ovary syndrome in a Tunisian women population. BMC Genomics. 2016;17(Suppl 9):748

23. Gomes MKDO, Antonino DDC, Balarin MAS, Tanaka SCSV, Caldeira MA, Trovó de Marqui $A B$, et al. Haplotype analysis of VEGF gene polymorphisms in polycystic ovary syndrome. Gynecol Endocrinol. 2019;35(10):847-50.
24. Guruvaiah P, Govatati S, Reddy TV, Lomada D, Deenadayal M, Shivaji S, et al. The VEGF +405 G>C 5' untranslated region polymorphism and risk of PCOS: a study in the south Indian women. J Assist Reprod Genet. 2014;31(10):1383-9.

25. Huang X, Hao YL, Zhen XL, Zhou RM, Wang N, Cao SR, et al. Association between the vascular endothelial growth factor gene polymorphisms and the risk of polycystic ovary syndrome in northern Chinese women. Gynecol Endocrinol. 2019:35(8):706-9.

26. Lee EJ, Oh B, Lee JY, Kimm K, Park JM, Baek KH. Association study between single nucleotide polymorphisms in the VEGF gene and polycystic ovary syndrome. Fertil Steril. 2008:89(6):1751-9.

27. Vural P, Kusku-Kiraz Z, Dogru-Abbasoglu S, Cil E, Karadag B, Akgul C, et al. Vascular endothelial growth factor-2578 A/C,-460 T/C and+405 $\mathrm{G} / \mathrm{C}$ polymorphisms in polycystic ovary syndrome. Eur J Obstetrics Gynecol Reprod Biol. 2009;147(1):57-60.

28. Ding $X$, Yin F. Gene single nucleotide polymorphisms of VEGF and the susceptibility for polycystic ovary syndrome. Progress Obstetrics Gynecol. 2009;18(11):805-8.

29. Li N, Yang Q, Zhao M. Aassociation between VEGF gene polymorphisms and polycystic ovary syndrome. China Foreign Med Treat. 2014;33(14): 94-6.

30. Rotterdam EA-SPcwg. Revised 2003 consensus on diagnostic criteria and long-term health risks related to polycystic ovary syndrome (PCOS). Hum Reprod. 2004;19(1):41-7.

31. Goodman NF, Cobin RH, Futterweit W, Glueck JS, Legro RS, Carmina E, et al. American association of clinical endocrinologists, American college of endocrinology, and androgen excess and Pcos society disease state clinical review: guide to the best practices in the evaluation and treatment of polycystic ovary syndrome - part 2. Endocrine Pract. 2015;21(12):1415-26.

32. Goodman NF, Cobin RH, Futterweit W, Glueck JS, Legro RS, Carmina E, et al. American association of clinical endocrinologists, American college of endocrinology, and androgen excess and Pcos society disease state clinical review: guide to the best practices in the evaluation and treatment of polycystic ovary syndrome--part 1. Endocrine Pract. 2015; 21(11):1291-300.

33. Hartling L, Milne A, Hamm MP, Vandermeer B, Ansari M, Tsertsvadze A, et al. Testing the Newcastle Ottawa scale showed low reliability between individual reviewers. J Clin Epidemiol. 2013:66(9):982-93.

34. Brok J, Thorlund K, Wetterslev J, Gluud C. Apparently conclusive meta-analyses may be inconclusive--trial sequential analysis adjustment of random error risk due to repetitive testing of accumulating data in apparently conclusive neonatal meta-analyses. Int J Epidemiol. 2009;38(1):287-98.

35. Tal R, Seifer DB, Arici A. The emerging role of angiogenic factor dysregulation in the pathogenesis of polycystic ovarian syndrome. Semin Reprod Med. 2015;33(3):195-207.

36. Thomas $J$, Eichmann A. The power of VEGF (vascular endothelial growth factor) family molecules. Cell Mol Life Sci. 2013;70(10):1673-4.

37. Hoeben A, Landuyt B, Highley MS, Wildiers $H$, Van Oosterom AT, De Bruijn EA. Vascular endothelial growth factor and angiogenesis. Pharmacol Rev. 2004;56(4):549-80.

38. Ferrara N. Vascular endothelial growth factor. Arterioscler Thromb Vasc Biol. 2009;29(6):789-91.

39. Ferrara N, Gerber HP. The role of vascular endothelial growth factor in angiogenesis. Acta Haematol. 2001;106(4):148-56.

40. Agrawal R, Jacobs H, Payne N, Conway G. Concentration of vascular endothelial growth factor released by cultured human luteinized granulosa cells is higher in women with polycystic ovaries than in women with normal ovaries. Fertil Steril. 2002;78(6):1164-9.

41. Renner W, Kotschan S, Hoffmann C, Obermayer-Pietsch B, Pilger E. A common $936 \mathrm{C} / \mathrm{T}$ mutation in the gene for vascular endothelial growth factor is associated with vascular endothelial growth factor plasma levels. J Vasc Res. 2000;37(6):443-8

42. Watson CJ, Webb NJ, Bottomley MJ, Brenchley PE. Identification of polymorphisms within the vascular endothelial growth factor (VEGF) gene: correlation with variation in VEGF protein production. Cytokine. 2000;12(8):1232-5.

43. Jung YW, Ahn EH, Kim JO, An HJ, Cho SH, Kim YR, et al. Association of genetic polymorphisms in VEGF $-460,-7$ and -583 and hematocrit level with the development of idiopathic recurrent pregnancy loss and a metaanalysis. J Gene Med. 2018;20(9):e3048. 
44. Su M, Hu Z, Dong C, Xu X. Vascular endothelial growth factor gene polymorphisms and hypertensive disorder of pregnancy: a metaanalysis. Pregnancy Hypertens. 2019;17:191-6.

45. Sun Y, Chen M, Mao B, Cheng X, Zhang X, Xu C. Association between vascular endothelial growth factor polymorphism and recurrent pregnancy loss: a systematic review and meta-analysis. Eur J Obstet Gynecol Reprod Biol. 2017;211:169-76.

\section{Publisher's Note}

Springer Nature remains neutral with regard to jurisdictional claims in published maps and institutional affiliations.

Ready to submit your research? Choose BMC and benefit from:

- fast, convenient online submission

- thorough peer review by experienced researchers in your field

- rapid publication on acceptance

- support for research data, including large and complex data types

- gold Open Access which fosters wider collaboration and increased citations

- maximum visibility for your research: over $100 \mathrm{M}$ website views per year

At $\mathrm{BMC}$, research is always in progress.

Learn more biomedcentral.com/submissions 